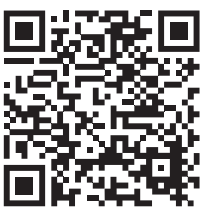

* Doctor en Pedagogía por la Universidad Nacional Autónoma de México.

Ciudad de México, México.

Correspondencia: $\mathrm{MRL}$ mreyna@unam.mx Conflicto de intereses: El autor declara no tener intereses personales, comerciales, financieros o económicos directos o indirectos, ni conflicto de intereses de cualquier índole que pudiese representar un sesgo para la información presentada en el artículo dictaminado. Citar como: Reyna LM. La ética en las políticas públicas y legales para la salud de los adolescentes (México). Rev CONAMED. 2020; 25(3): 146-151. https:// dx.doi.org/10.35366/95986

Financiamiento: Ninguno.

Recibido: 14/08/2020

Aceptado: 08/09/2020

\section{La ética en las políticas públicas y legales para la salud de los adolescentes (México)}

\author{
Ethics in public and legal policies for the health of adolescents (México) \\ Mauricio Reyna Lara*
}

\section{RESUMEN}

El artículo tiene como objetivo fortalecer los valores éticos de la salud en el adolescente al mostrar la evidencia reciente sobre el estudio publicado por la Organización Mundial de la Salud (OMS) con respecto a la inactividad física, la obesidad y el sobrepeso en los adolescentes; las cuales propician las enfermedades no transmisibles (ENT) como una de las causas de muerte en el mundo; asimismo, este escrito expone mayormente el caso de México, a pesar de que el problema es a nivel mundial. La programación y proyección de políticas públicas, su evaluación y seguimiento, además de propiciar iniciativas legales sobre la salud de los adolescentes, mejoran su calidad de vida y garantizan que los gobiernos cumplan con los compromisos internacionales en la Agenda 2030.

Palabras clave: Salud en el adolescente, inactividad física, obesidad y sobrepeso de los adolescentes.

\section{ABSTRACT}

The article aims to strengthen the ethical values of health in adolescents, showing recent evidence on the study published by the World Health Organization (WHO) regarding the physical inactivity, obesity and weight of adolescents; What causes noncommunicable diseases (NCDs) as one of the causes of death in the world, refers mostly to the case of Mexico, although the health problem is worldwide. The programming and projection of public policies, their evaluation and monitoring, in addition to promoting legal initiatives on adolescent health, improves their quality of life and ensures that governments comply with international commitments in the 2030 Agenda.

Keywords: Adolescent health, physical inactivity, obesity and overweight of adolescents.

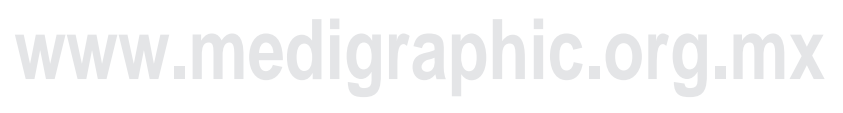




\section{INTRODUCCIÓN}

Debemos contemplar ante todo lo descrito en la Constitución Mundial de la Salud: «La salud de todos los pueblos es una condición fundamental para lograr la paz y la seguridad, y depende de la más amplia cooperación de las personas y de los Estados». Tomando en consideración que ante las omisiones o acciones contrarias a la salud se atenta contra la dignidad humana. ${ }^{2}$ La Organización Mundial de la Salud (OMS) reportaba en $2010^{3}$ recomendaciones mundiales sobre la actividad física para la salud, ya que la inactividad física era el cuarto factor de riesgo de mortalidad y prevalencia de enfermedades no transmisibles (ENT) en el mundo; para ello se tomó en consideración una escala mundial de varios países y los estudios realizados que mostraban que 81\% de los adolescentes de 11 a 17 años de edad no se presentaban activos. Las mujeres se observaban con menor actividad de un $78 \%$, mientras que los hombres representaban un 84\%. Dentro de los motivos se observaban la urbanización que conlleva a los factores de inseguridad, los largos trayectos de movilidad, la calidad de contaminación en el aire y la falta de instalaciones que motivan el deporte, tal como infraestructuras de parques y deportivos.

La Organización Mundial de la Salud creó instrumentos que fungieron como guía para poder determinar los avances y seguimientos de los niveles de actividad física (Global School-Based Student Health Survey: CSHS) y el plan de acción mundial para la prevención y el control de las enfermedades no transmisibles 2013-2020. Asimismo «La OMS define la actividad física como cualquier movimiento corporal producido por los músculos esqueléticos, con el consiguiente consumo de energía. Ello incluye las actividades realizadas al trabajar, jugar y viajar, las tareas domésticas y las actividades recreativas». ${ }^{4}$

El artículo pretende difundir el reciente estudio (2019) de la Organización Mundial de la Salud con la idea de identificar el problema por país, para la formulación de posibles soluciones y la selección de alternativas que permitan su seguimiento y evaluación en la salud de los adolescentes; esto con énfasis en la ética de la política pública y con acciones del Estado en la sociedad, que permitan a los lectores adolescentes provocar conciencia en que no existirá un sistema de salud que pueda abatir los padecimientos futuros o presentes de quienes no puedan cuidar de su salud. El estudio se focaliza en los adolescentes en México como referente; sin embargo, puede ser útil en algunos otros países de Iberoamérica y el mundo a quienes podamos concientizar con las buenas prácticas en la decisión de políticas públicas en salud de las autoridades públicas responsables para los adolescentes, con la idea de reducir enfermedades no trasmisibles con la activación física para evitar la obesidad y el sobrepeso.

\section{Análisis actual en el mundo y para México}

El último estudio de la Organización Mundial de la Salud publicado en 2019 con datos sobre la inactividad física de los adolescentes fue en la revista The Lancet Child \& Adolescent Health. ${ }^{5}$ La estimación de la referencia en jóvenes de 11 a 17 años de edad llegó a representar $77.6 \%$ de hombres y $84 \%$ de mujeres que no tienen actividad física, tomando en consideración que la encuesta fue enfocada a 146 países a estudiantes a nivel mundial con datos encuestados en el año 2016. La variable que se observó en el estudio publicado por la OMS fue que, conforme al nivel socioeconómico y potencial de ingreso por país, presentaba una variante: los países con bajos recursos de ingreso la incidencia fue de 79.3\%, en los países con recursos de ingreso medio presente fue de $83.9 \%$ y para los de ingresos altos fue de $79.4 \%$. Para México, la inactividad física en general de los adolescentes representó $83.2 \%$, de los cuales $78.8 \%$ son hombres y $87.6 \%$ son mujeres.

Es necesario contemplar los datos de la Encuesta Nacional de Salud y Nutrición (ENSANUT) 2018; 6 los datos de la encuesta o levantamiento de la información fueron del 30 de julio de 2018 al 28 de junio de 2019, el tamaño fue dividida por el tema del acceso a los servicios de salud, un muestreo de 50,000 viviendas y por el tema nutricional un promedio de 32,000 viviendas, lo que en promedio estimaron representar a 126.5 millones de habitantes de México. Según la misma encuesta, se consultaron 22.8 millones de jóvenes entre 10 y 19 años, los cuales presentaron sobrepeso y obesidad de 12 a 19 años, con una alimentación baja nutricionalmente. Los gobiernos deben dar continuidad 
a los programas y proyectos que reduzcan dicho problema de salud, más aún con el reciente estudio publicado por la Organización Mundial de la Salud, sin importar la transición de cambio de sus gobiernos para dirigir la administración pública. En el libro coordinado por Rolando F. y otros, titulado Política y políticas públicas en los procesos de reforma de América Latina, el autor que interviene en uno de los artículos, Yves Surel, señala que «Todo actor político que llega al poder se encuentra, en efecto, frente a un conjunto de programas y de proyectos lanzados por sus predecesores, que él deberá asumir o reformar». ${ }^{7}$

La mala alimentación asociada con una inactividad física de los jóvenes nos inserta en la preocupación por establecer políticas públicas transversales de los gobiernos que reduzcan la obesidad y el sobrepeso de los adolescentes, ya que de lo contrario potencializa padecimientos multifactoriales en la salud de su vida presente y futura en la población. Cortina ${ }^{8}$ ha señalado que las instituciones de salud deben asumir sus acciones profesionales con una responsabilidad social y bajo un criterio ético de su formación frente a la atención de la salud pública de la población. La Comisión Nacional de los Derechos Humanos en México publicó, el 22 de octubre de 2019 , la recomendación general 39/20199 sobre los derechos de los niños, niñas y adolescentes ante el incremento de sobrepeso y obesidad infantil y de adolescentes, con la idea de erradicar consumos excesivos de alimentos y bebidas con altos contenidos energéticos.

\section{Estrategias éticas para una política pública}

La política pública en salud visualiza acciones éticas necesarias y prioritarias para los adolescentes, dada la evidencia que ha sido presentada por la OMS y los gobiernos de diferentes países, dentro de las cuales deben contemplarse:

- Es necesario establecer de manera permanente la difusión, con el contenido de información sobre la buena alimentación nutritiva en los adolescentes en escuelas y espacios públicos.

- Estimar el valor consciente y ético del conocimiento sobre la calidad de los alimentos y el resultado en la salud.
- No puede seguirse omitiendo la información del valor nutricional de los alimentos, lo que propicia obligar a las autoridades el poder atender la accesibilidad de los alimentos.

- Fomentar comedores comunitarios y universitarios es una tarea de inversión necesaria para la política pública de los gobiernos, en donde el monitoreo de la autoridad sanitaria sea con la intención de cuidar la calidad de la dieta ofertada para los comensales por el restaurantero. La Universidad Nacional Autónoma de México ha podido contribuir con dicha dinámica a su interior con los alumnos al establecer Becas de Apoyo Nutricional para que los estudiantes que tengan accesibilidad a los comedores universitarios en las facultades y escuelas ${ }^{10}$ sean alimentos de calidad, esto ha sido una de las acciones encaminadas a reducir el problema, sin embargo, no se ha podido señalar que ello se replique actualmente para todo el país.

- Debe incentivarse a la iniciativa privada con el estímulo fiscal, para los productores de alimentos con ingredientes orgánicos, que evite encarecer el producto para los consumidores.

- El estímulo económico laboral por control de peso y masa corporal en los adolescentes que se encuentran activos en el entorno laboral, hará que mejore su productividad y eficiencia en el desarrollo de sus actividades.

- La apertura de horarios y prestaciones para el cuidado de su actividad física cerca o en su área de trabajo.

- Motivar actividades de participación en el deporte y convertirlo en una contraprestación que permite mejorar su calidad de vida. No puede ser que hasta la actualidad el ir al gimnasio es visto como una actividad de lujo y no como un complemento en la forma de vida para la salud, es más, no es ni deducible de impuesto, siendo un servicio de los que se hacen uso para beneficio de la salud y que puedan servir como una devolución al impuesto en México.

- Acrecentar las becas y los programas sociales que contribuyan a la permanencia de sus estudios y desarrollar actividades deportivas y culturales.

- No puede focalizarse esfuerzos en apoyar deportistas de alto rendimiento y olvidar aquellos que puedan incorporar su vida diaria con la 
actividad deportiva. El deporte debe ser visto como un complemento a la formación integral de los adolescentes y no como un accesorio complementario. De la misma forma ha venido contribuyendo la Universidad Nacional Autónoma de México como ejemplo de ello, al tener más de 42 modalidades de becas para sus alumnos y alumnas. Para identificar la dimensión de la población estudiantil a la que nos referimos, tenemos que en ciclo escolar 20182019 se inscribieron un total de 356,530 alumnos y alumnas, en su mayoría adolescentes, en los niveles educativos de bachillerato, licenciatura y postgrado, cursos propedéuticos y técnicos profesionales; de los cuales en las diversas modalidad de becas y apoyos se becaron a más de 200 mil estudiantes," recursos que contribuyen a la permanencia de los estudiosy a una debida alimentación.

- El tiempo de transportación que se utiliza hoy en día en las grandes ciudades es excesivo, puede ir desde 40 minutos hasta tres horas o más al día, lo que ocasiona un grave problema a la salud, debe motivarse acciones y estrategias que reduzcan la movilidad de los adolescentes, sobre todo en las grandes ciudades como la Ciudad de México; reduciendo los tiempos de movilidad se mejoran las formas de vida diaria y el medio ambiente. Existen trabajos en el sector público y privado que pueden tener una proyección para desarrollar sus actividades desde casa, midiendo sus actividades mediante indicadores de productividad, sin la presencia de los adolescentes incorporados en la vida laboral de manera física en los lugares de trabajo, la vida a distancia mediante las Tecnologías de la Información y Comunicación (TIC) son una realidad en nuestros días.

- Es necesario una mayor supervisión en los productos comercializados que mantengan la calidad del producto y no se altere su costo. La variedad de productos que se ponen a la venta en tiendas de autoservicio, además de contener el etiquetado de los ingredientes, es necesario verificar sus contenidos, no sólo en la autorización para comercializarlo sino con posteridad.

- Si legislativamente, desde la Constitución Política de los Estados Unidos Mexicanos, se establece en su artículo 4 el derecho a la alimentación de calidad y nutritiva; ¿por qué el consumidor debe pagar más por un mejor producto y no exigir de las autoridades sanitarias a los productores que los productos comercializados sean de calidad y a buen precio? Los gobiernos deben garantizarlo con su autorización y supervisión el derecho a la alimentación con calidad.

- Debe crearse un organismo administrativo denominado policía sanitaria que se encuentre dotado de conocimiento interdisciplinario y multidisciplinario en el área de la salud, con la especialización del derecho sanitario y de bioética, con la idea de poder darle competencia de supervisar todo establecimiento de productos perecederos o no perecederos, industrializadosy para consumo humano, que se han patentado y autorizado para su comercialización al interior de los países. Dicha vigilancia permitiría garantizar la permanencia del producto de calidad en el mercado y el mejoramiento de la salud de sus consumidores, como es el caso de los establecimientos de comida rápida o la cadena de restaurantes, así como las tiendas de autoservicio como supermercados. Se debe transformar la producción de comida chatarra a comida nutritiva por los mismos productores y consumidores. La regla es que los adolescentes y la ciudadanía en general como consumidores, identifiquen los ingredientes del producto y la utilidad nutritiva que le produce en su consumo, no sólo por la descripción de la etiqueta, que ya existe una ley que obliga a describirlo (en México), sino por la reacción física o mental en el consumo de los ingredientes del producto.

- El uso desmedido de videojuegos mediante las diversas tecnologías de información y comunicación, en las escuelas y con las familias, provoca una inactividad física y revela la necesidad de su regulación jurídica, al ser calificado como un padecimiento en las adicciones de los adolescentes, según lo indicado por la Organización Mundial de la Salud.

\section{Propuestas legislativas con orientación biotecnológica}

La biotecnología hoy en día es un sin número de posibilidades para la industrialización de los alimentos y la salud. Las leyes se han quedado aletargadas ante los grandes descubrimientos de 
la ciencia, la industrialización de los alimentos cada vez se vuelve un tema fundamental en la vida diaria y en la exigencia de las necesidades de la sociedad por alimentarse; sin embargo, no existe una debida supervisión en la producción permanente de los alimentos, ni mucho menos la exigencia por la bioseguridad de ciertos productos no regulados o no controlados. La certificación del producto orgánico es un medio de control bajo estándares internacionales (Organización de las Naciones Unidas para la Alimentación) y nacionales que otorga mayor confiabilidad del producto, sin embargo, encarece el producto y disminuye su accesibilidad para el consumidor.

El comer bien representa un costo que repercute en el nivel socioeconómico de la población y eso no puede seguirse comercializando de esa forma, ya existen nuevas formas de poder implementar el desarrollo y la potencialidad de la agricultura. La tecnología digital, en bienes públicos globales de inversión que empodere a los pequeños, medianos y grandes empresarios en el ámbito de la alimentación y agricultura, se denomina por la Organización para la Alimentación y la Agricultura (FAO) la ciberagricultura. ${ }^{12}$

Uno de los grandes retos de los indicadores de la agenda 2030 es el desarrollo sostenible de la agricultura y la seguridad alimentaria, basado en mejorar la salud de la población, de ahí que sea muy importante atender y evaluar las políticas públicas del gobierno mexicano sobre el tema; asimismo, robustecer el contenido en las legislaciones que encaminen la obligación del sector privado y público para poder producir mayores alimentos de calidad.

La inversión de programas y proyectos de la agricultura debe ser mayor, la gran urbanización ha olvidado resguardar las tierras que eran fuente y factor de producción agrícola, la mancha urbana va incorporando nuevas viviendasy queda en segundo término la inversión en el campo, la discusión ética sobre la agricultura y la alimentación es indispensable para el futuro de la salud, ya sea tanto en alimentación como en la producción de medicamentos. ${ }^{13}$

\section{CONCLUSIONES}

- El año 2020 se caracterizará en México y en el mundo por la perturbación que deja la pandemia por el virus SARS-CoV-2 en la sociedad, ${ }^{14}$ se incrementan los problemas en la salud de la ciudadanía, al generar la atención en los servicios de salud, para contemplar la emergencia sanitaria, al acordar el Consejo de Salubridad General. Medidas extraordinarias en el territorio nacional el día 30 de marzo de 2020, para reducir la propagación del virus, nos llevan a realizar todas nuestras actividades desde casa, siendo una medida para contener el contagio del virus; no obstante, se incrementan los problemas de salud en los adolescentes, como es el caso de la obesidad y el sobrepeso por la inactividad.

- Las estrategias de las políticas públicas de salud y sobre temas diversos transversales en la administración pública del Gobierno de México, deben ir orientadas a poder activar a los adolescentes, mejorando y previniendo su estado de salud físico y mental; el seguimiento y evaluación de los proyectos que orienten a resolver el problema debe ser una preocupación preponderante en nuestros días en el Sistema Nacional de Salud.

- Los resultados obtenidos en las investigaciones de la Organización Mundial de la Salud, así como de los compromisos internacionales como son la Declaración de Incheon ${ }^{75}$ y sus objetivos de educación para dotar a los niños, niñas y adolescentes del conocimiento necesario para vivir dignamente, son referentes que contextualizan la idea de políticas públicas educativas bajo contenidos para prevenir su estado de salud, siendo además un marco de acción para reportar la agenda 2030, con el objetivo de garantizar una vida sana y promover el bienestar para todos en todas la edades, y en específico sobre el rubro de grupos vulnerables y retos de la universalidad en los servicios de salud para adolescentes.

- Con políticas públicas éticas, basadas en evidencias que reduzcan los rezagos en la salud de los adolescentes con el modelo de competencias en una educación formal e informal, que contribuyan con el entorno y la vida sustentable, lo que ayudará a mejorar su vida y la de futuras generaciones.

- Cambiar los patrones legislativos en materia laboral y administrativos para facilitar los trámites de servicios, escuelas y trabajos a distancia, 
utilizando las tecnologías de información y comunicación para simplificar la movilidad de los adolescentes en las zonas urbanas y rurales.

- Una plena vigilancia de las autorizaciones, permisos y patentes de la industrialización de los alimentos, de tal forma que exista de manera permanente la vigilancia mediante la competencia de cuerpos especializados en el ámbito sanitario, con el carácter multidisciplinario e interdisciplinarios, capaces de poder detectar los productos en los establecimientos de venta y de consumo, como es el caso de contar con los conocimientos de especialización en derecho sanitario y bioética.

- Motivar la inversión pública y privada de la ciberagricultura para mejorar y agilizar la calidad de productos en el campo.

- Vincular las necesidades administrativas de operación a los legisladores, que permitan actualizar los enunciados normativos de la ley a la implementación de políticas públicas en salud de actualidad en el beneficio y desarrollo de los adolescentes, conforme su interacción y necesidad actual de la sociedad.

- La especificación de estrategias que vayan dirigidas en particular a los adolescentes de 11 a 17 años de edad con la activación física corporal, involucrando medidas didácticas mediante juegos que puedan ser más atractivos para la permanencia y continuidad de las actividades en los adolescentes.

- La educación física como una actividad integral de las familias y escuelas, como un mínimo de 60 minutos diarios.

- Y, en concreto, desarrollar políticas públicas que alcancen el monitoreo cualitativo y cuantitativo de las recomendaciones para el fomento de la actividad física y así mejorar la salud y prevenir las enfermedades no transmisibles (ENT).

\section{Bibliografía}

1. Constitución de la Organización Mundial de la Salud. Principios básicos. [Consultada 22/01/2020] Disponible en: http://apps.who.int/gb/bd/PDF/bd48/basic-documents48th-edition-sp.pdf\#page=7
2. Cortina A. Justicia cordial. Madrid, España: Editorial Trotta;2010.

3. Recomendaciones mundiales sobre la actividad física para la Salud de la Organización Mundial de la Salud. 2010. [Fecha de consulta 10/12/2019] Disponible en: https://apps.who.int/iris/ bitstream/handle/10665/44441/9789243599977_spa.pdf?ua=1

4. Organización Mundial de la Salud. Actividad física. 2018. [Fecha de consulta 10/12/2019] Disponible en: https://www. who.int/es/news-room/fact-sheets/detail/physical-activity

5. Tendencias mundiales en la actividad física insuficiente entre los adolescentes. [Fecha de consulta 09/12/2019] Disponible en: https://www.thelancet.com/journals/lanchi/ article/PIIS2352-4642(19)30323-2/fulltext

6. INEGI Encuesta Nacional de la Dinámica Demográfica 2018. Base de datos. SNIEC. Información de Interés Nacional. México: [Fecha de consulta 09/12/2019]. Disponible en: https:// uww.inegi.org.mx/contenidos/programas/ensanut/2018/doc/ ensanut_2018_presentacion_resultados.pdf

7. Rolando F, Lanzaro J. Política y políticas públicas en los procesos de reforma de América Latina. Madrid, España: Miño y Dávila Editores; 2006.

8. Cortina A. Conferencia: ética de las instituciones de salud. Universidad del Desarrollo. Centro de Bioética. 2003.

9. Comisión Nacional de Derechos Humanos. [Consultado 22/01/2020] Disponible en: file:///C:/Users/Mauricio/ Downloads/417-2019.pdf

10. Universidad Nacional Autónoma de México. Portal del Becario de la UNAM. [Consulta 22/01/2020] Disponible en: https://www.becarios.unam.mx/Portal2018/?page_id=1969

11. Universidad Nacional Autónoma de México, Agenda Estadística 2019. [Consultada 22/01/2020] Disponible en: https://www.planeacion.unam.mx/Agenda/2019/pdf/ Agenda2019.pdf

12. Organización de las Naciones Unidas para la Alimentación y la Agricultura. Guía de estrategias de agricultura electrónica. [Consulta 22/01/2020] Disponible en: http:// www.fao.org/in-action/e-agriculture-strategy-guide/en/

13. Valls R. La dignidad humana. En: Casado M (coord.). Sobre la dignidad y los principios. Análisis de la Declaración Universal sobre Bioética y Derechos Humanos, UNESCO. 2009. Cizur Menor, Civitas; pp. 401-410.

14. Acuerdo por el que se establecen acciones extraordinarias para atender la emergencia sanitaria generada por el virus SARS-Cov2, Diario Oficial de la Federación, consulta realizada por la página web:: https://n9.cl/nlog Fecha de Consulta 07/10/2020).

15. Organización de las Naciones Unidas para la Educación, la Ciencia y la Cultura Educación 2030: Declaración de Incheon y Marco de Acción para la realización del Objetivo de Desarrollo Sostenible: https://unesdoc.unesco.org/ ark:/48223/pf0000245656_spa 